\title{
The influence of the signal-to-noise ratio upon radio occultation inversion quality
}

\author{
Michael Gorbunov ${ }^{1,2,3}$ \\ ${ }^{1}$ A.M. Obukhov Institute of Atmospheric Physics, Russian Academy of Sciences, Pyzhevsky per. 3, Moscow, 119017, Russia \\ ${ }^{2}$ Hydrometcenter of Russia, Bolshoy Predtechensky per. 11-13, Moscow, 123242, Russia \\ ${ }^{3}$ Spire Global Inc., 1825 33rd St, Boulder, CO 80301
}

Correspondence: Michael Gorbunov (gorbunov@ifaran.ru)

\begin{abstract}
In this paper, we investigate the influence of the signal-to-noise ratio (SNR) upon the radio occultation (RO) retrieval quality. We perform two series of numerical simulations: 1) with artificial RO data and, 2) with real COSMIC observations. We superimpose the simulated white noise with varying magnitudes upon both types of the observation data and evaluate the response in the statistics. The statistics use the reference fields of the analyses of European Centre for Medium-Range Weather

5 Forecasts (ECMWF). Our simulations indicate that the effect of additive white noise has a threshold character: the influence of the noise is very low up to some threshold, but when the threshold is exceeded, the influence increases dramatically. Another conclusion is that, given RO observations of fair quality, the enhancement of the SNR cannot be expected to provide significant improvement in retrieval quality.
\end{abstract}

\section{Introduction}

In this study, we investigate the influence of the signal-to-noise ratio (SNR) of radio occultation (RO) signals upon the retrieval quality. The SNR is a unitless variable that characterizes the magnitude of the electromagnetic field recorded by the spaceborne receiver mounted on the Low Earth Orbiter (LEO). SNR is measured in V/V and is understood as the ratio of the useful signal and the internal receiver noise.

The concept of noise in RO observation is broader than just that related to SNR. The quality of neutral atmospheric retrieval is affected by the residual ionospheric noise, which is not included in SNR. The ionospheric component of the excess phase can be treated as noise in the neutral atmospheric retrieval, but it is the useful signal when considering ionospheric retrieval. SNR, being based on the intrinsic receiver niose figure, has never been treated as the useful signal.

The neutral atmospheric retrieval quality has two different aspects: 1) quality of individual profiles, 2) statistical properties of large arrays of retrieved profiles. Interesting examples of deep occultations in the presence of pronounced humidity layers are discussed by Sokolovskiy et al. (2014). For measuring such events, it is important to have a high SNR because signals observed deep below the planet limb, correspond to large and sharp spikes in the bending angle profile and are, therefore, weak. Such events may be statistically insignificant for numerical weather prediction (NWP) purposes, still, they are interesting for the study of the planetary boundary layer (PBL) (Sokolovskiy et al., 2006). 
When proposing new RO missions, like COSMIC-2 (launched and now beginning an operational phase) (Sokolovskiy et al., 2019) or the high-gain instrument proposed by PlanetiQ (Kursinski, 2019), an improved SNR is treated as an essential advantage and is expected to result in an improved retrieval quality in the troposphere.

In this study, we analyze the influence of additive white noise upon the RO retrieval quality. To this end, we perform numerical simulations with analyses of European Center for Medium-Range Weather Forecasts (ECMWF), where we superimpose random noise on artificial RO data and analyze the influence of the noise magnitude on the retrieval quality, characterized by mean and random mean square deviation. A second part of the study uses COSMIC data, where we superimpose the random noise upon the COSMIC RO observations and analyze the influence of the noise magnitude upon the comparison of retrieved refractivity profiles with the reference ECMWF data.

\section{Noise model}

In this study, we assume a model of the observed complex signal with additive noise:

$\hat{u}_{m}(t)=u_{m}(t)+\xi_{m}(t)$

where $u_{m}(t)$ is the true complex signal in the $m$-th channel, and $\xi_{m}(t)$ is the complex random noise. The true signal can also be represented by the amplitude and excess phase:

$u_{m}(t)=A_{m}(t) \exp \left(i k\left(\Psi_{0}(t)+\Delta \Psi_{m}(t)\right)\right)$,

where $A_{m}(t)$ is the true amplitude of the signal in the $m$-th channel, $\Psi_{0}(t)$ is the satellite-to-satellite distance, and $\Delta \Psi_{m}$ is the true excess phase in the $m$-th channel.

Noise $\xi_{m}(t)$ is assumed to be white, independent for different channels. White noise is a generalized random process that is characterized by a flat spectral distribution of its energy $\Phi_{m}(\xi)=\Phi_{m}=$ const, which implies an infinite dispersion. It is understood as a generalized derivative of the Brownian motion (Hida, 1980).

Given discrete moments of time $t_{j}$, we can consider a random uncorrelated process, $\hat{\xi}_{m}\left(t_{j}\right)$, which equals $\xi_{m}(t)$ averaged over time intervals $\Delta t=t_{j+1}-t_{j}$. It is straightforward to evaluate its dispersion:

$$
\begin{aligned}
\left\langle\left|\hat{\xi}_{m}\left(t_{j}\right)\right|^{2}\right\rangle & =\frac{1}{\Delta t^{2}} \int_{0}^{\Delta t} \int_{0}^{\Delta t}\left\langle\xi_{m}\left(t^{\prime}\right) \xi_{m}^{*}\left(t^{\prime \prime}\right)\right\rangle d t^{\prime} d t^{\prime \prime} \\
& \left.=\frac{1}{\Delta t^{2}} \int_{0}^{\Delta t} \int_{0}^{\Delta t} \iint \tilde{\xi}_{m}\left(\omega^{\prime}\right) \tilde{\xi}_{m}^{*}\left(\omega^{\prime \prime}\right)\right\rangle \exp \left(i \xi^{\prime} t^{\prime}-i \xi^{\prime \prime} t^{\prime \prime}\right) d \omega^{\prime} d \omega^{\prime \prime} d t^{\prime} d t^{\prime \prime}
\end{aligned}
$$

where $\tilde{\xi}_{m}(\omega)$ is the generalized Fourier transform of $\xi_{m}(t)$. For a stationary random process, we have the following relationship (Rytov et al., 1988):

$50\left\langle\tilde{\xi}_{m}\left(\omega^{\prime}\right) \tilde{\xi}_{m}^{*}\left(\omega^{\prime \prime}\right)\right\rangle=\Phi_{m}\left(\omega^{\prime}\right) \delta\left(\omega^{\prime}-\omega^{\prime \prime}\right)$. 
This allows transforming (3) as follows:

$$
\begin{aligned}
\left\langle\left|\hat{\xi}_{m}\left(t_{j}\right)\right|^{2}\right\rangle & =\frac{1}{\Delta t^{2}} \int_{0}^{\Delta t} \int_{0}^{\Delta t} \int_{0} \Phi_{m}(\omega) \exp \left(i \omega\left(t^{\prime}-t^{\prime \prime}\right)\right) d \omega d t^{\prime} d t^{\prime \prime}= \\
& =\frac{2 \pi}{\Delta t^{2}} \Phi_{m} \int_{0}^{\Delta t} \int_{0}^{\Delta t} \delta\left(t^{\prime}-t^{\prime \prime}\right) d t^{\prime} d t^{\prime \prime}=\frac{2 \pi}{\Delta t} \Phi_{m}=2 \pi f_{s} \Phi_{m}
\end{aligned}
$$

where $f_{s}$ is the sampling rate. This relation indicates that the white noise with discrete time occupies the band width of $2 \pi f_{s}$ in terms of angular frequency, or $f_{s}$ in terms of ordinary frequency. The relative strength of the fluctuations is evaluated as follows:

$$
\frac{\left\langle\left|\hat{\xi}_{m}\left(t_{j}\right)\right|^{2}\right\rangle}{\left\langle A_{m}^{2}(t)\right\rangle}=f_{s} \frac{2 \pi \Phi_{m}}{\left\langle A_{m}^{2}(t)\right\rangle}=f_{s} \widehat{\Phi}_{m}
$$

where we use the normalized spectral density $\widehat{\Phi}_{m}$ measured in the unit of the inverse sampling frequency. The signal-to-noise spectral density $C N_{0}$ is measured in $\mathrm{dB}-\mathrm{Hz}$ and defined as follows:

$60 C N_{0}=-10 \lg \left(2 \hat{\Phi}_{m}\right)$,

where the factor of 2 takes into account the fact that the double-sided spectrum is used. Accordingly, the noise magnitude is evaluated as follows:

$$
\left\langle\left|\hat{\xi}_{m}\left(t_{j}\right)\right|^{2}\right\rangle=\frac{f_{s}\left\langle A_{m}^{2}(t)\right\rangle}{2 \times 10^{0.1 C N_{0}}}
$$

\section{Numerical Simulations}

\subsection{Artificial data with superimposed noise}

In the numerical simulations, we used the analyses of the European Center for Medium-Range Weather Forecasts as the reference truth. We generated artificial RO data using the Wave Optics Propagator (WOP) package based on the split-step, or phase-screen technique (Martin, 1992; Gorbunov, 2002). Upon simulated RO data, we superimposed noise with different magnitudes. We generated artificial data using the geometry from COSMIC events observed on January 1, 2008. Figures 1 and 2 show the mean and RMS differences of the retrieved and reference refractivity. We used signal-to-noise levels with a step of $10 \mathrm{~dB}-\mathrm{Hz}$, from 67 to 27 . Relative noise levels $\left(\left\langle\left|\hat{\xi}_{m}\right|^{2}\right\rangle /\left\langle A_{m}^{2}(t)\right\rangle\right)^{1 / 2}$ according to Eq. (8) are presented in Table 1 . Numerical simulations were also conducted for higher SNR up to $127 \mathrm{~dB}-\mathrm{Hz}$. However, they indicated that the effect of the white noise is vanishingly small.

Figure 3 shows the comparison of the WOP data with superimposed noise (denoted as "WN" in the figures) with reference ECMWF data (denoted as "E" in the figures) and reference WOP data without noise (denoted as "W" in the figures). The RMS and mean refractivity difference at heights of $z=5 \mathrm{~km}$ and $z=20 \mathrm{~km}$ are plotted as a function of the noise level $C N_{0}$. 
2008. WOP - ECMWF. World

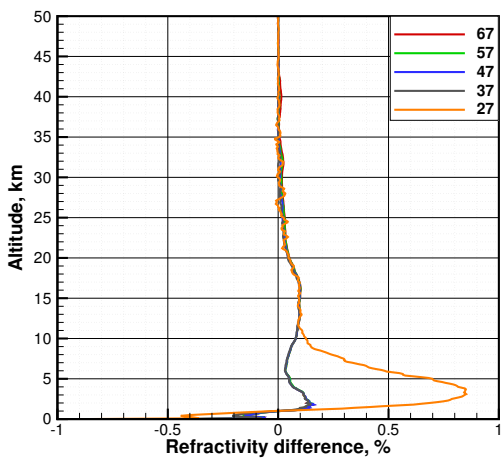

2008. WOP - ECMWF. 30-60

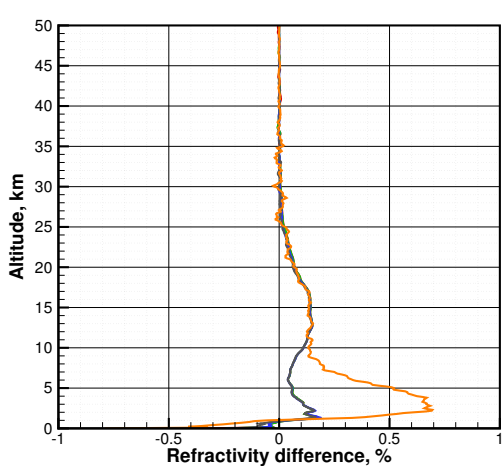

2008. WOP - ECMWF. 0-30

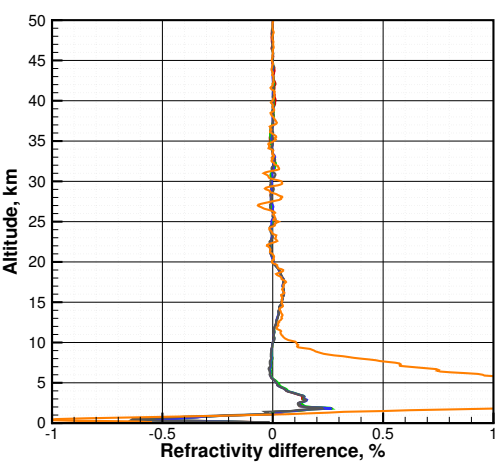

2008. WOP - ECMWF. $60-90$

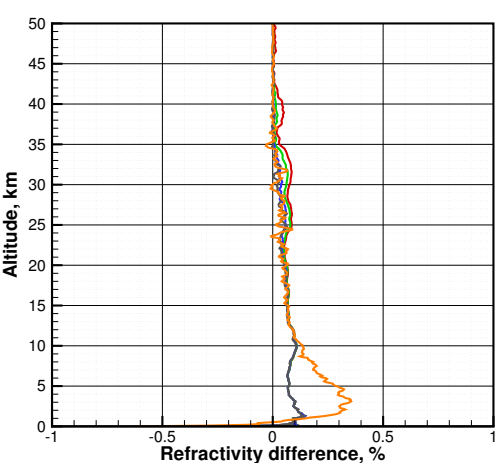

Figure 1. Numerical simulation with ECMWF data. Mean relative difference between the retrieved and reference refractivities for different signal-to-noise levels: 67, 57, 47, 37, and $27 \mathrm{~dB}-\mathrm{Hz}$. The four panels show the regionlaized statistics. World: The whole globe. 0-30: latitudes $0^{\circ}-30^{\circ}$. 30-60: latitudes $30^{\circ}-60^{\circ} .60-90$ : latitudes $60^{\circ}-90^{\circ}$. The latitude bands include both South and North hemispheres. Note that large mean realtive differences between the retrieved and reference refractivities are evident only for a low SNR of $27 \mathrm{~dB}-\mathrm{Hz}$.

Table 1. Relation between $C N 0$, relative noise level, and SNR [V/V].

\begin{tabular}{|l|l|l|}
\hline$C N_{0}, \mathrm{~dB}-\mathrm{Hz}$ & Noise level, relative & $\mathrm{SNR}, \mathrm{V} / \mathrm{V}$ \\
\hline 67 & 0.0022 & 454 \\
\hline 57 & 0.0071 & 140 \\
\hline 47 & 0.022 & 45 \\
\hline 37 & 0.071 & 14 \\
\hline 27 & 0.22 & 4.5 \\
\hline 17 & 0.71 & 1.4 \\
\hline
\end{tabular}

These results indicate that the influence of the white noise has a threshold effect. For $C N_{0}>27 \mathrm{~dB}-\mathrm{Hz}$, the effect of white noise is hardly noticeable. Still, the WN-W difference plotted in the logarithmic scale (Figure 3) indicates that the noise 
2008. WOP - ECMWF. World

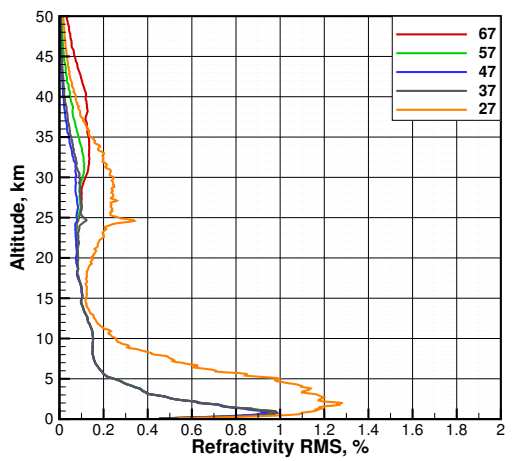

2008. WOP - ECMWF. 30-60

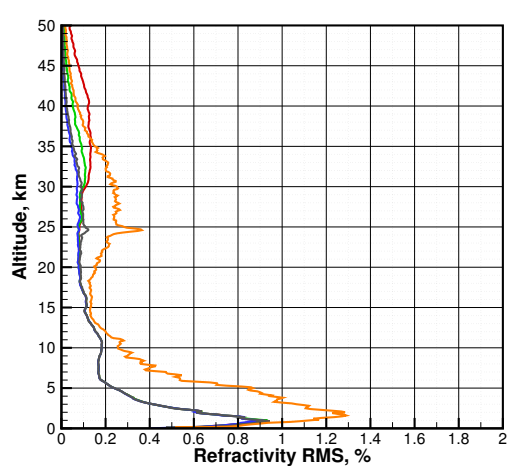

2008. WOP - ECMWF. 0-30

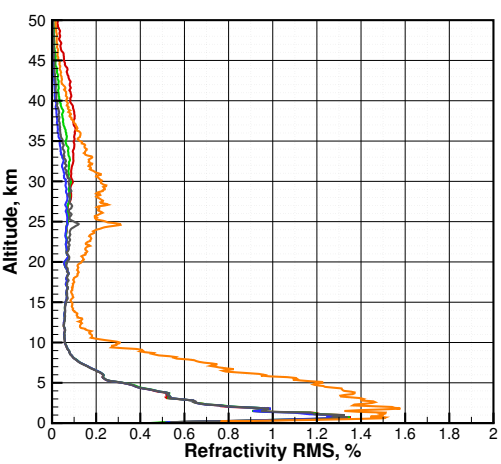

2008. WOP - ECMWF. $60-90$

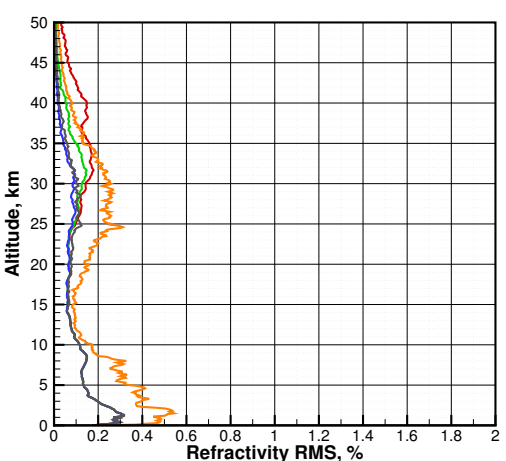

Figure 2. Numerical simulation with ECMWF data. RMS relative difference between the retrieved and reference refractivities for different noise levels: 67, 57, 47, 37, and $27 \mathrm{~dB}-\mathrm{Hz}$. Panels are the same as in Figure 1

influence increases approximately linearly with respect to the noise amplitude. At the height of $z=20 \mathrm{~km}$, the critical noise level corresponds to $C N_{0}=27 \mathrm{~dB}-\mathrm{Hz}$, and for $z=5 \mathrm{~km}$ it corresponds to $C N_{0}=32 \mathrm{~dB}-\mathrm{Hz}$. When the noise threshold is exceeded, its influence dramatically increases, resulting also in large systematic errors and the decrease of data that pass the quality control (QC).

\subsection{COSMIC Data with Superimposed Noise}

We preformed another series of experiments with COSMIC data. The ECMWF analyses were again used as the reference. We processed one day of COSMIC events from January 1, 2008. We superimposed noise with the same levels $C N_{0}$, as those used in the simulations with the artificial data, and checked how this influenced the statistical comparison with ECMWF, as well as how much this perturbed the inversion. Here we also present results for $C N_{0}$ up to $67 \mathrm{~dB}-\mathrm{Hz}$, because, like for the artificial data, the effect of white noise for higher SNR is vanishingly small. 

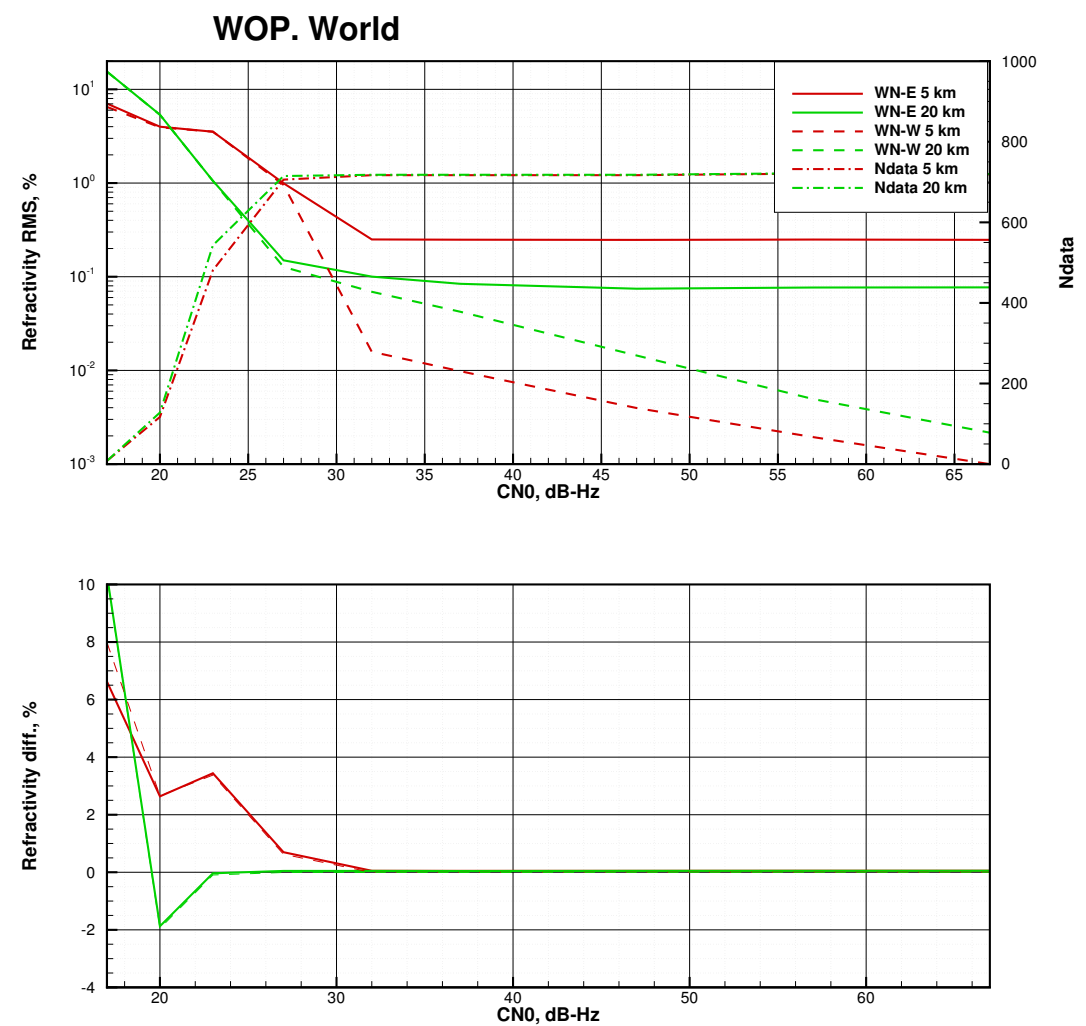

Figure 3. The RMS and mean difference between the reference and retrieved refractivities for ECMWF-based simulations, at heights of $z=5 \mathrm{~km}$ (red curves) and $z=20 \mathrm{~km}$ (green curves) as function of noise level. Notation: "W" is the reference WOP data without noise; "WN" is the WOP data with superimposed noise; "E" is the reference ECMWF data; "Ndata" is the number of data.

Figures 4 and 5 show, respectively, the bias and RMS of the refractivities retrieved from COSMIC with different noise levels. Figures 6 and 7 show, respectively, the bias and the RMS of refractivities retrieved from COSMIC with superimposed noise with respect to those retrieved from the unperturbed COSMIC data.

Figure 8 shows the RMS and mean refractivity differences at heights of $z=5 \mathrm{~km}$ and $z=20 \mathrm{~km}$ as a function of the noise level $C N_{0}$. Here we see that the noise effect becomes large for $C N_{0} \lesssim 37 \mathrm{~dB}-\mathrm{Hz}$. For the height of $z=20 \mathrm{~km}$, these results are very similar to the WOP simulation. The noise threshold is also $C N_{0}=27 \mathrm{~dB}-\mathrm{Hz}$. However, for the height $z=5 \mathrm{~km}$, the sensitivity to noise is higher, and the threshold is about $C N_{0}=32 \mathrm{~dB}-\mathrm{Hz}$. This can be explained by the fact that lower tropospheric data are influenced by much stronger inherent fluctuations that can also be interpreted as multiplicative self-noise (Gorbunov et al., 2015). 
2008. COSMIC - ECMWF. World

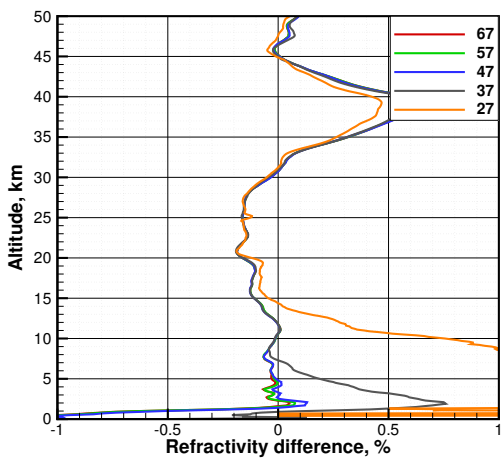

2008. COSMIC - ECMWF. 30-60

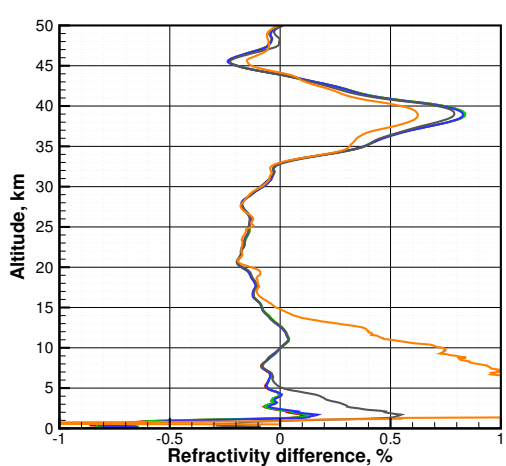

2008. COSMIC - ECMWF. 0-30

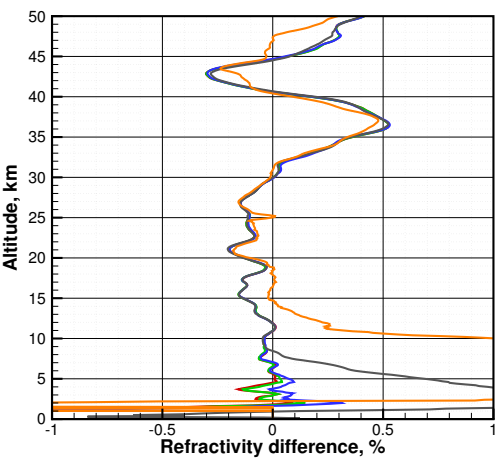

2008. COSMIC - ECMWF. $60-90$

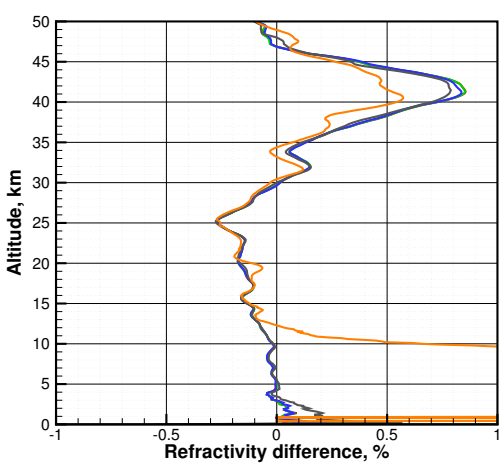

Figure 4. Comparison of processing COSMIC data with superimposed noise with ECMWF data. Mean relative difference between the retrieved and reference refractivities for different noise levels: 67, 57, 47, 37, and $27 \mathrm{~dB}-\mathrm{Hz}$. Panels are the same as in Figure 1

\section{Conclusions}

In this study, we investigated the influence of random measurement noise upon the statistical quality of RO data inversion. To this end, we performed a series of numerical simulations with artificial RO data. The simulations indicated that the effect of additive white noise has very much a threshold effect. For values of $C N_{0}>27 \div 32 \mathrm{~dB}-\mathrm{Hz}$, the effect of the noise is very low and does not influence the retrieval quality significantly. For WOP data, the noise threshold is independent from height, while for COSMIC data the lower-tropospheric data indicate stronger, noise-like fluctuations and, therefore, a higher value of the threshold $C N_{0}=32 \mathrm{~dB}-\mathrm{Hz}$. For weaker SNRs, e.g., when $C N_{0} \lesssim 17 \mathrm{~dB}-\mathrm{Hz}$, the data become unusable.

We conclude that an RO mission exceeding the SNR thresholds noted here will provide good data, and any improvement in the SNR cannot be expected to significantly improve the resulting statistics. This also indicates that RO missions based on smaller satellite platorms such as CubeSats and without high gain antennas will not inherently suffer poor retrieval quality if their minimum SNR exceeds these thresholds. Here, we only studied the statistics of the neutral refractivity retrieval. The 
https://doi.org/10.5194/amt-2020-114

Preprint. Discussion started: 22 April 2020

(C) Author(s) 2020. CC BY 4.0 License.

(c) (1)

\section{Atmospheric Measurement \\ Techniques \\ Discussions}

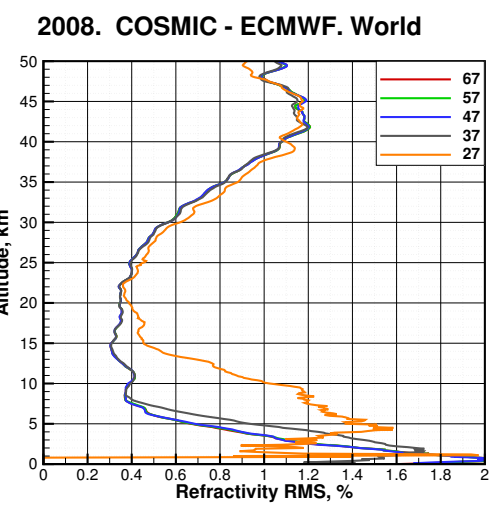

2008. COSMIC - ECMWF. 30-60

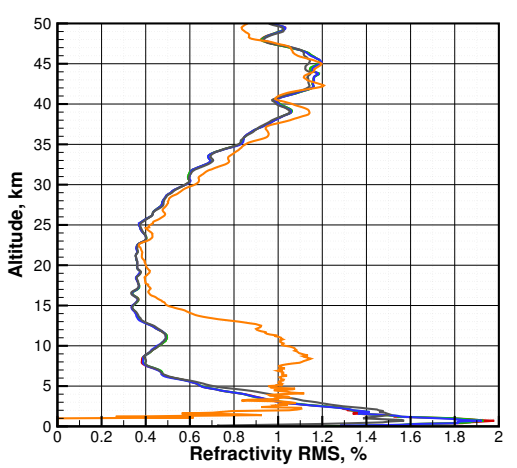

2008. COSMIC - ECMWF. 0-30

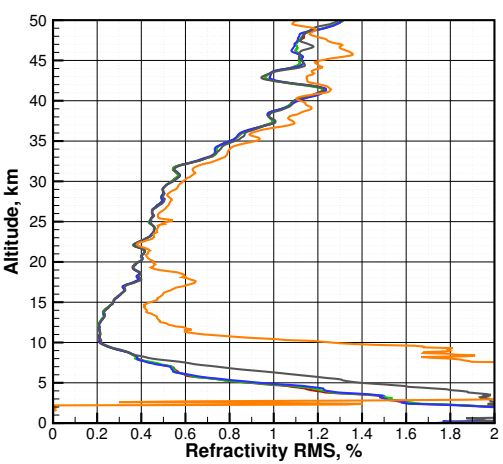

2008. COSMIC - ECMWF. $60-90$

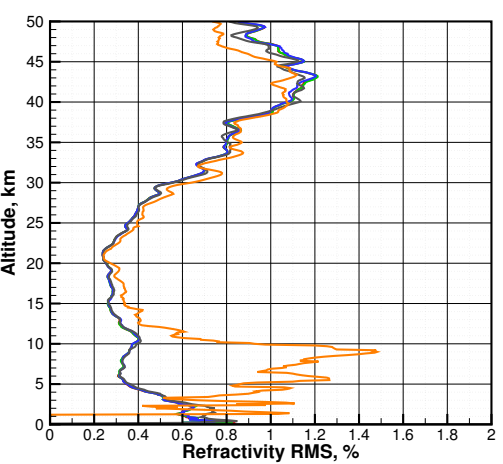

Figure 5. Comparison of processing COSMIC data with superimposed noise with ECMWF data. RMS relative difference between the retrieved and reference refractivities for different noise levels: 67, 57, 47, 37, and $27 \mathrm{~dB}-\mathrm{Hz}$. Panels are the same as in Figure 1

influence of the noise level on the processing of deep, PBL RO signals revealing intensive humidity stratification and other features more appropriate for research and less consequential for NWP applications, requires an additional study.

Competing interests. I declare that no competing interests are present.

Acknowledgements. This study was supported by Russian Foundation for Basic Research (grant No. 20-05-00189 A). Author is grateful to C. Rocken (GPS Solutions) for valuable discussions. 
2008. COSMIC_N - COSMIC. World

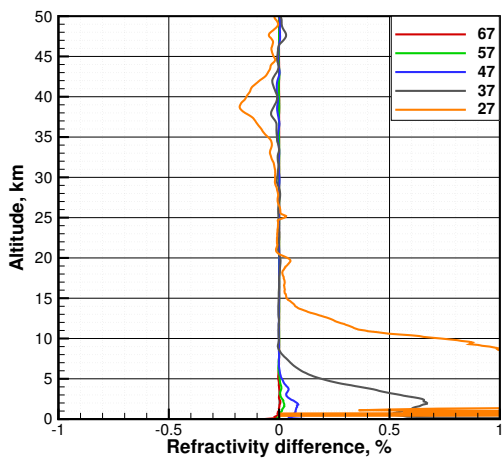

2008. COSMIC_N - COSMIC. $30-60$

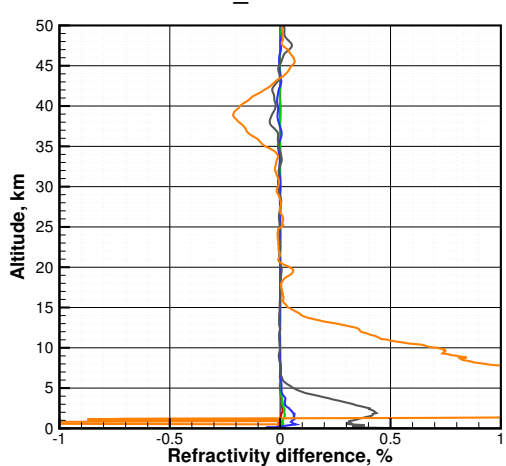

2008. COSMIC_N - COSMIC. $\mathbf{0 - 3 0}$

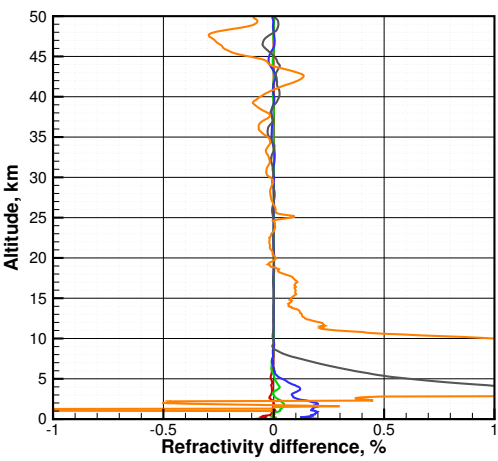

2008. COSMIC_N - COSMIC. $60-90$

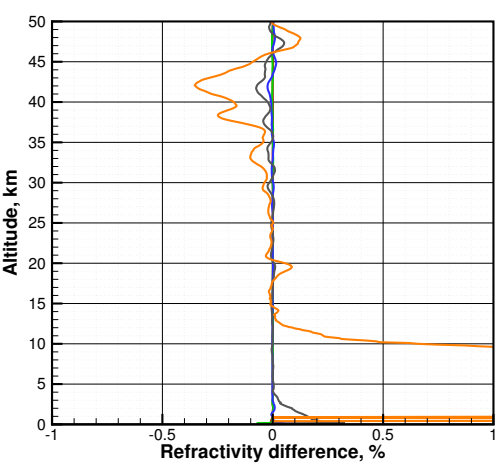

Figure 6. Comparison of processing COSMIC data with superimposed noise with original COSMIC data. Mean relative difference between the retrieved and reference refractivities for different noise levels: 67, 57, 47, 37, and $27 \mathrm{~dB}-\mathrm{Hz}$. Panels are the same as in Figure 1

\section{References}

Gorbunov, M. E.: Canonical transform method for processing radio occultation data in the lower troposphere, Radio Sci., 37, 9-1-9-10, https://doi.org/10.1029/2000RS002592, 2002.

Gorbunov, M. E., Vorob'ev, V. V., and Lauritsen, K. B.: Fluctuations of refractivity as a systematic error source in radio occultations, Radio Sci., 50, 656-669, https://doi.org/10.1002/2014RS005639, 2015.

Hida, T.: Brownian Motion, Springer US, https://doi.org/10.1007/978-1-4612-6030-1, 1980.

Kursinski, E. R.: Weather \& Space Weather RO Data from PlanetiQ Commercial GNSS RO, in: Joint 6th ROM SAF Data User Workshop and 7th IROWG Workshop, 19-25 September 2019, Konventum, Elsinore, Denmark, https://www.romsaf.org/romsaf-irowg-2019/en/ open/1570200998.1d65c4b7ee0ec946ef8ea65b0d718d2d.pdf/Kursinski_PlanetiQ+Overview+IROWG+091919.pdf, 2019.

Martin, J.: Simulation of wave propagation in random media: theory and applications, in: Wave propagation in random media (scintillations), edited by Tatarskii, V. I., Ishimaru, A., and Zavorotny, V. U., pp. 463-486, SPIE - The International Society for Optical Engineering and Institute of Physics Publishing, Bellingham, Washington USA, Bristol and Philadelphia, 1992. 
https://doi.org/10.5194/amt-2020-114

Preprint. Discussion started: 22 April 2020

(C) Author(s) 2020. CC BY 4.0 License.

(c) (i)
Atmospheric

Measurement

Techniques

Discussions
2008. COSMIC_N - COSMIC. World

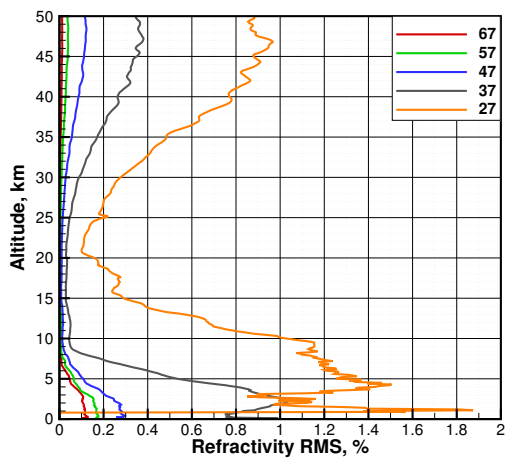

2008. COSMIC_N - COSMIC. $30-60$

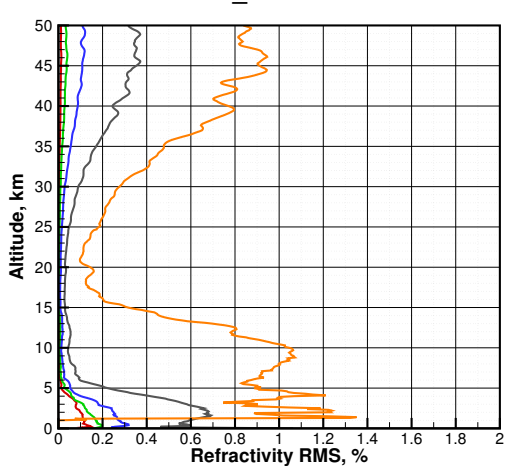

2008. COSMIC_N - COSMIC. $\mathbf{0 - 3 0}$

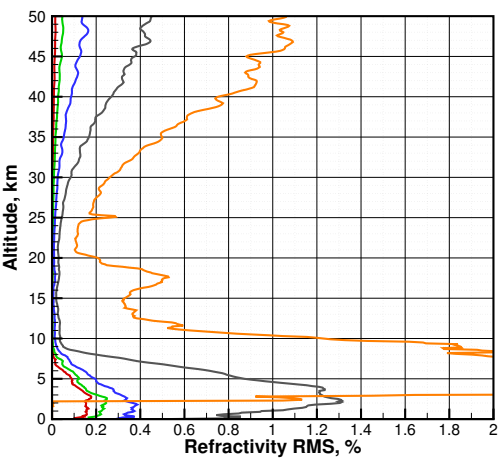

2008. COSMIC_N - COSMIC. $60-90$

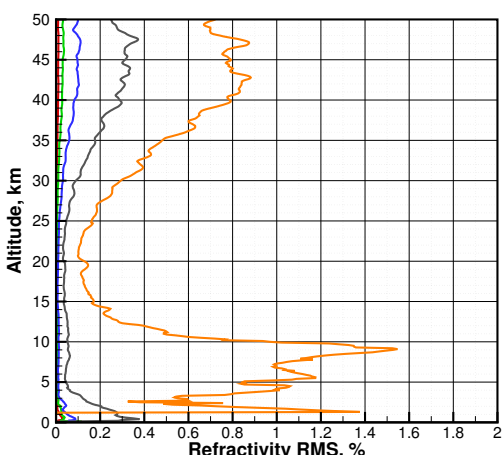

Figure 7. Comparison of processing COSMIC data with superimposed noise with original COSMIC data. Mean relative difference between the retrieved and reference refractivities for different noise levels: 67, 57, 47, 37, and $27 \mathrm{~dB}-\mathrm{Hz}$. Panels are the same as in Figure 1

Rytov, S. M., Kravtsov, Y. A., and Tatarskii, V.: Principles of Statistical Radiophysics : Correlation Theory of Random Processes Vol 2, Springer-Verlag, Berlin Heidelberg, 1988.

Sokolovskiy, S., Rocken, C., Hunt, D., Schreiner, W., Johnson, J., Masters, D., and Esterhuizen, S.: GPS profiling of the lower troposphere from space: Inversion and demodulation of the open-loop radio occultation signals, Geophys. Res. Lett., 33, L14816, https://doi.org/10.1029/2006GL026112, 2006.

Sokolovskiy, S., Schreiner, W., Zeng, Z., Hunt, D., Lin, Y.-C., and Kuo, Y.-H.: Observation, analysis, and modeling of deep radio occultation signals: Effects of tropospheric ducts and interfering signals, Radio Sci., 49, 954-970, https://doi.org/10.1002/2014RS005436, 2014.

Sokolovskiy, S., Schreiner, W., Weiss, J., Zeng, Z., Hunt, D., and Braun, J.: Initial Assessment of COSMIC-2 Data in the Lower Troposphere, in: Joint 6th ROM SAF Data User Workshop and 7th IROWG Workshop, 19-25 September 2019, Konventum, Elsinore, Denmark, https://www.romsaf.org/romsaf-irowg-2019/en/open/1570202428.cf195c6aa62789cfb81f06d529ecdf0f.pdf/ Sokolovskiy_IROWG-UCAR-C2-LT_Final.pdf, 2019. 
https://doi.org/10.5194/amt-2020-114

Preprint. Discussion started: 22 April 2020

(C) Author(s) 2020. CC BY 4.0 License.

\section{Atmospheric Measurement \\ Techniques \\ Discussions}
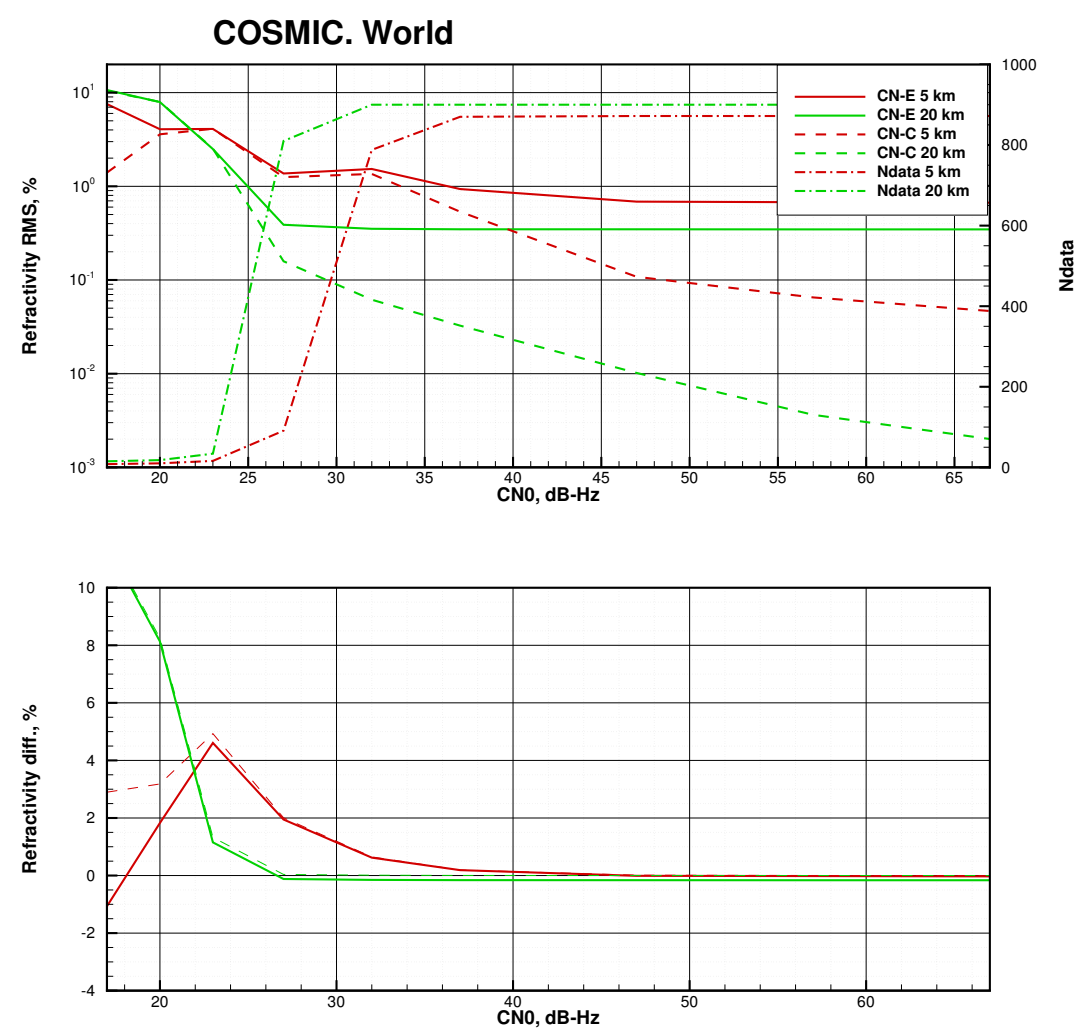

Figure 8. The RMS and mean difference between the reference and retrieved refractivities for ECMWF-based simulations, at heights of $z=5 \mathrm{~km}$ (red curves) and $z=20 \mathrm{~km}$ (green curves) as function of noise level. Notation: C - reference COSMIC data without superimposing additional noise; CN - COSMIC data with superimposed noise; E - reference ECMWF data; Ndata - number of data. 